\title{
Reconstructive surgery is effective for recurrent kneecap dislocation
}

\section{Brandon Erickson \\ Joseph Nguyen \\ Katelyn Gasik \\ Simone Gruber \\ Jacqueline Brady \\ Beth E. Shubin Stein}

\section{Video Abstract}

Keywords: American Journal of Sports Medicine, Hospital for Special Surgery, Rothman Institute, medial patellofemoral ligament reconstruction (MPFL), patellar instability, knee, tibial tubercle trochlear groove (TT-TG) distance, outcome, surgery, patellar height, bony anatomy, Knee Injury and Osteoarthritis Outcome Score Quality of Life, return to sport (RTS), dislocation, patellar stabilization, recurrent dislocation, tibial tubercle osteotomy (TTO), patient-reported outcome measure (PROM), trochlear dysplasia, radiography

Posted Date: September 20th, 2019

DOI: https://doi.org/10.21203/rs.2.14992/v1

License: (c) (i) This work is licensed under a Creative Commons Attribution 4.0 International License. Read Full License 


\section{Abstract}

New research suggests that for young patients who experience recurring dislocation of the kneecap, reconstructive surgery is an effective treatment option. Among 90 physically active patients undergoing surgery, nearly all were able to return to the court or field in just under 9 months on average, with fewer than a handful reporting recurring instability. Lateral patellar instability is a common problem affecting patients aged 10 to 25 . Rehab without surgery is the current standard of care for those undergoing patellar dislocation for the first time. But for repeat sufferers, the path to recovery is less clear. While there is some consensus on taking the surgical route for ligament repair, recommendations on additional bone repair are mixed. On top of the variety of anatomical criteria for making that decision, there's the possibility that adding such treatment to the surgical menu could increase the risk of complication. To provide some clarity, researchers from the Hospital for Special Surgery in New York followed 90 patients with a history of patellar dislocation who underwent reconstruction of the medial patellofemoral ligament -regardless of bony anatomy. Their results showed that patients improved significantly across nearly all patientreported outcomes measured. At 1 year after surgery, $96 \%$ of patients reported no recurrent instability. And after two years, $100 \%$ reported no recurrence. Additionally, $90 \%$ of patients reported a return to sports at 1 year, and $88 \%$ at 2 years-with an average return time of 8.8 months. Only two patients reported partial or full redislocation. Notably, no patient underwent any bone restructuring, suggesting that favorable outcomes with a less extensive procedure are possible. The research team cites a few limitations of their work. First, the results apply mostly to the short period following surgery; although the team followed patients for up to 4 years after treatment, most reported on their outcomes only within the first two years. Also, the study comes from a single high-volume surgeon with a special interest in the patellofemoral joint. The results, therefore, may not translate to all surgeons. Still, the results are encouraging. For the thousands of patients and doctors wading through various options, the teams' findings could help illuminate the best path to recovery. 